\title{
Experimental Testing on Phantom Image for Improved MRI Compressed Sensing
}

\author{
Aleksandar Kamilovski
}

\begin{abstract}
This paper presents a possible way for improving the techniques of compressed sensing and parallel imaging techniques for brain MRI. Experimental tests have been performed over a phantom test image. An exclusive elliptical sampling mask has been generated, which in combination with double-density wavelet transforms offers improvement over the standard approach. Additional tests undertaken as part of this research propose the usage of nonlinear reconstruction method, generated elliptical sampling mask and double-density wavelet transform for application of compressed sensing to brain MRI. An assessment of the results for diagnostic usage has been done by a specialist of radiology.

Keywords - Brain MRI, phantom test image, compressed sensing, parallel imaging, sampling patterns, wavelet transform, double-density wavelets, POCS, zero-filled, GRAPPA.
\end{abstract}

\section{INTRODUCTION}

$\mathrm{T}$ HE MRI scanning process is tedious, considering the fact, that it, in general, takes between 15 and 40 minutes. The region has survived from high enthusiasm over the previous decade. The focus is on reducing the time of scans, which embroils more patients to be served every day in medical centers. The challenge is observed in several directions. This paper introduces the outcome of the research in direction for improving the techniques of compressed sensing and parallel imaging by choosing an efficient wavelet transformation paired with an appropriate sampling mask. The most recent research discoveries [1][5], give a strong foundation to believe that research in this direction can result in improved algorithms of compressed sensing [6]. Lustig et al [1] - [2] describe the basics of compressed sensing and highlight the performance dependency out of the sampling pattern choice and used transformation in MRI. Wajer et al in [3] give a detailed explanation for improving the sampling schemes. Evladov et al, [4], present a method based on Radon transform. In [5] Weizmann et al use reference information from the initial scan. The technique is based on different slices from the same scan of the patient and the goal is to achieve an adapted and efficient subsampling scheme.

Paper received June 17, 2019; revised December 22, 2019; accepted December 23, 2019. Date of publication July 31, 2020. The associate editor coordinating the review of this manuscript and approving it for publication was Prof. Branimir Reljin.

This paper is revised and expanded version of the paper presented at the 26th Telecommunications Forum TELFOR 2018 [9].

Aleksandar Kamilovski is with Keitaro DOO, Bul. Partizanski Odredi 14, 1000 Skopje, Macedonia; (phone: 38978260274; e-mail: krusty362472@yahoo.com).
In our examination, the code was written and executed in Matlab R2015a. A primary tool for the experimental part and the results presented in this paper is software package SPIRiT V0.3 (SPIRiT: Iterative Self-Consistent Parallel Imaging Reconstruction from Arbitrary k-Space Sampling) [7]. The reference results are the ones that represent default settings from this software package. The source code of this package has been changed to test various wavelet transformations and sampling masks for improvement over the reference results. Software package VDrad (Variable Density sampling and Radial view-ordering) [8] has been used for generation of sets of sampling patterns, which were imported in software package SPIRiT, in order to find the most efficient sampling pattern for MRI brain scanning.

In section II of this paper reference results are presented obtained by usage of the default sampling mask and orthogonal wavelet transform applied to a phantom test image. In section III the experimental results are presented, obtained using the new generated sampling mask and various types of wavelet families. Section IV explains the results and concludes the research.

This paper is an addition to the published and reviewed paper presented at the 26th Telecommunications Forum TELFOR 2018[9]. Tests are expanded on a phantom test image and complement the initial experiment results.

\section{REFERENCE TESTS AND RESULTS}

The following steps are taken to test the efficiency of different wavelet transformations and sampling masks. The procedure starts by choosing the sampling pattern for compressed sensing of the MRI scan in k-space. The next step is normalization of the parameters and calibration of coils for the parallel imaging of multi-channel scans. The non-linear reconstruction technique is applied using the chosen wavelet transform and decomposition level and the result is a two-dimensional reconstructed MRI image. Considering the importance of reconstruction method for the quality of the reconstructed image, several reconstruction techniques are examined in the first phase of the testing. The goal is to determine the reconstruction method that will be the most suitable for this application. Visual comparison between the resulting reconstructed images for different techniques and normalized difference of the images is enabled. Image quality is followed by these measures: PSNR (Peak Signal to Noise Ratio), MAE (Maximum Absolute Error), SSIM (Structural Similarity Index Measure) and EPI (Edge Preservation Index). The choice to follow these parameters is based on similar research efforts, [6].

Initially, an eight-channel phantom image (phantom_8ch.mat) is reconstructed $(220 \times 160 \times 8)$, given as 
an example image in the SPIRiT package. Used input parameters are: number of iterations - 20, orthogonal wavelet - Daubechies 4, level of decomposition -4 and sampling mask - "rndm" shown in Fig. 1, also provided in the SPIRiT package. The following reconstruction methods were tested: POCS (Projection On Convex Sets), zero-filled and GRAPPA (GeneRalized Autocalibrating Partial Parallel Acquisition). Table 1 presents the quality measures of the reconstructed images, which are shown in Fig. 2. The best quality of the reconstructed image is achieved using POCS, which is the reason to choose POCS as a reconstruction method for further experiments.

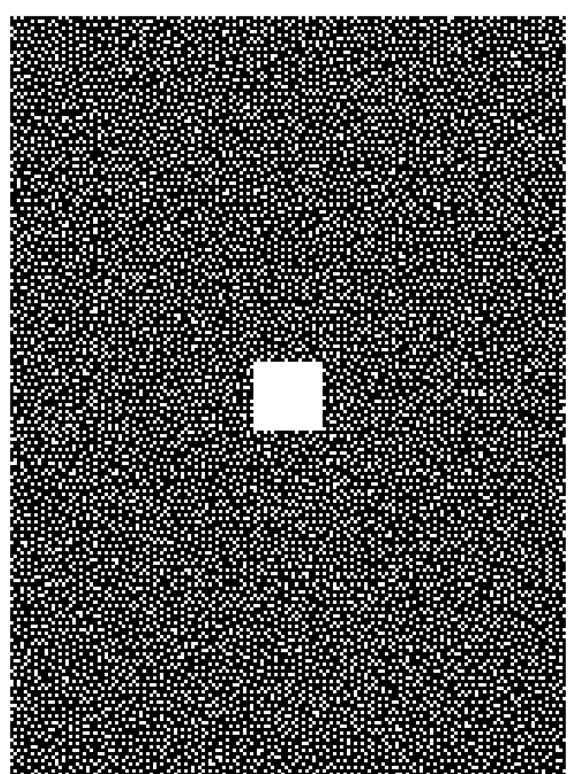

Fig. 1. Default sampling mask - "rndm".

Interesting information that needs to be highlighted is the number of samples taken with sampling mask, which is 9360 in case of "rndm". That is around $27 \%$ of the total number of samples of the original image. This implies that the acquisition will be almost 4 times faster compared to the case of normal acquisition of all the samples from the image.

TABLE 1: RECONSTRUCTION RESULTS USING "RNDM" MASK

\begin{tabular}{|c|c|c|c|c|}
\hline "rndm" & MAE & PSNR[dB] & SSIM & EPI \\
\hline POCS & 0.1590 & 30.6849 & 0.6939 & 0.9893 \\
\hline $\begin{array}{c}\text { zero-fill } \\
\text { dc }\end{array}$ & 0.5438 & 15.9501 & 0.2479 & 0.8874 \\
\hline $\begin{array}{c}\text { zero- } \\
\text { filled }\end{array}$ & 0.5291 & 20.2504 & 0.4103 & 0.9121 \\
\hline
\end{tabular}

\section{MODIFICATIONS OF THE TECHNIQUE}

The first step towards a better performance through modifications of the technique is generation of a new sampling mask. For this purpose, software package VDRad (Variable Density sampling and Radial view-ordering) was used, which provides a possibility for generation of a number of elliptical, square and triangle sample masks and their variations. This package is already proven and used for clinical diagnosis approved by medical experts. One of the successful applications of the masks generated from this package is in scanning of the abdominal parts of children. During the tests around 50 sampling masks were generated.
The best results, based on the quality measures, were achieved using the mask shown in Fig. 3.

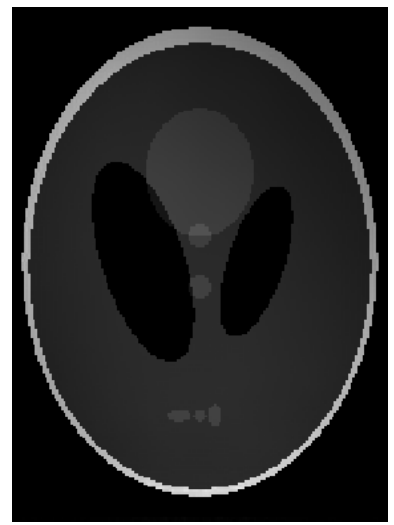

a)

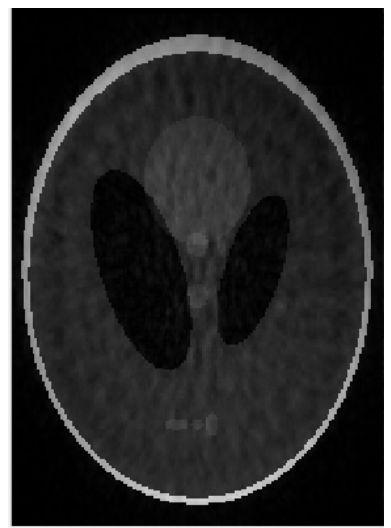

c)

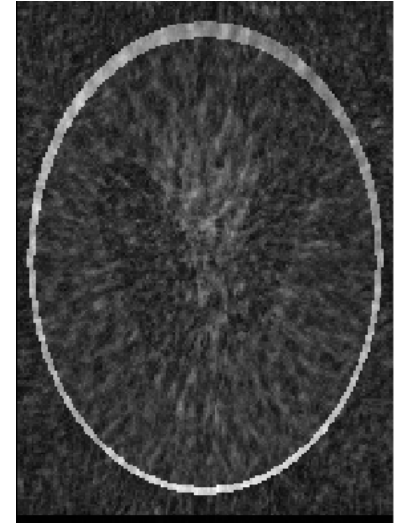

b)

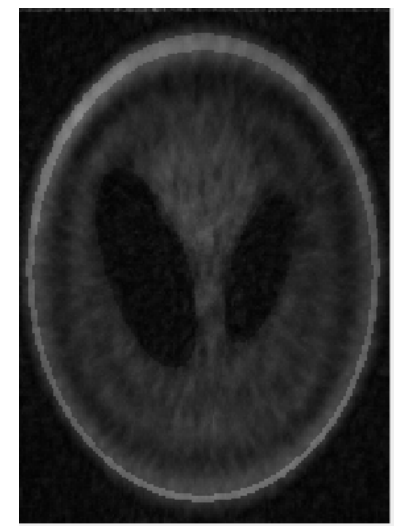

d)
Fig. 2. Reconstructed images using "rndm" mask: a) original; b) zero-filled d/c; c) POCS; d) zero-filled.

The number of samples taken with this mask is 6848 , which is around $19 \%$ of the total number of samples. That results in over five times faster acquisition and scanning. Using the sampling mask "phantom" the acquisition will be faster compared to "rndm" sampling mask because it has $73 \%$ of the number of samples of "random3" mask.

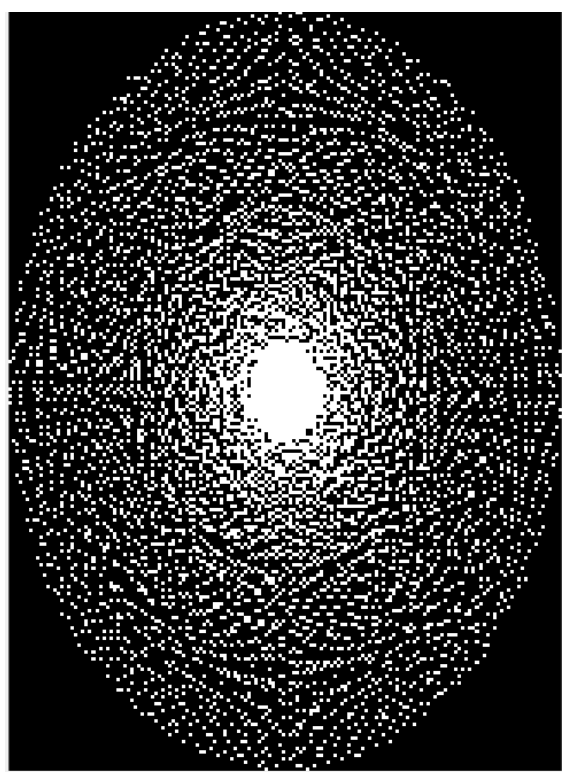

Fig. 3. Generated sampling mask - "phantom". 
Using the sampling mask "phantom", tests were performed with orthogonal wavelets of: Haar, Beylkin, Coiflet(n), Daubechies(n), Symmlet(n), Vaidyanathan, Battle(n), Lemarie and Pollen, including their translation invariant versions (total 134 variants), also biorthogonal wavelets: Triangle, Interpolating, Deslauriers, AverageInterpolating, $\mathrm{CDF}(\mathrm{n})$ and Villasenor(n) (total 39 variants of biorthogonal wavelets) and 15 additional variants of reverse-biorthogonal wavelets. This was the second step of modification. The best results per wavelet family are depicted in Table 2.

TABLE 2: RECONSTRUCTION RESULTS USING “PHANTOM" MASK

\begin{tabular}{|c|c|c|c|c|}
\hline "phantom" & MAE & PSNR[dB] & SSIM & EPI \\
\hline Orthogonal & 0.1750 & 34.7072 & 0.8935 & 0.9637 \\
\hline TI & 0.1779 & 34.9954 & 0.9109 & 0.9674 \\
\hline Biorthogonal & 0.1816 & 34.7072 & 0.8935 & 0.9637 \\
\hline $\begin{array}{c}\text { Reverse- } \\
\text { biorthogonal }\end{array}$ & 0.1846 & 34.7072 & 0.8935 & 0.9637 \\
\hline
\end{tabular}

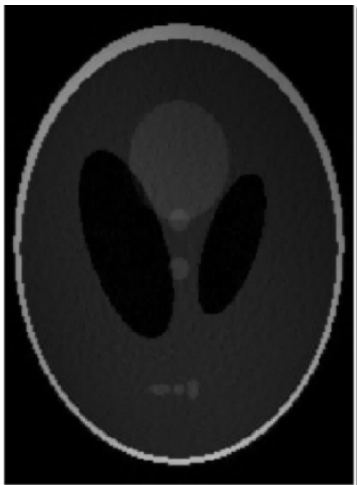

a)

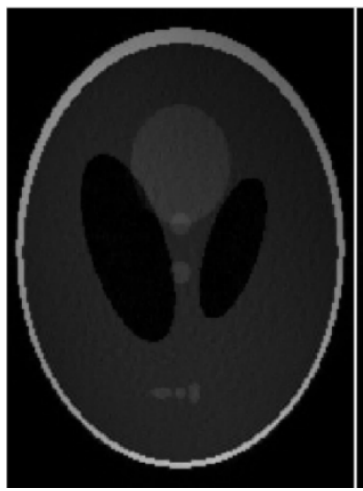

c)

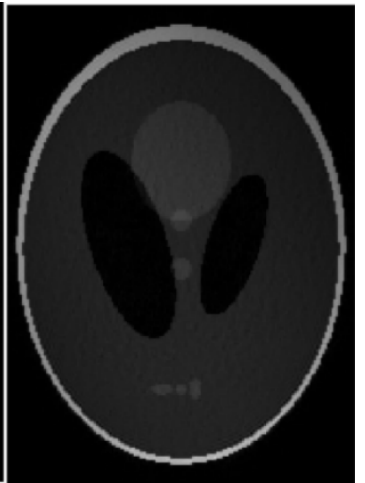

b)

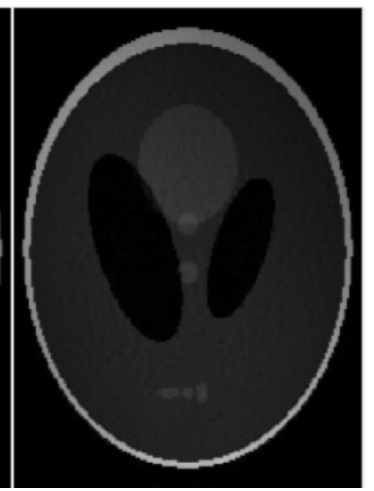

d)
Fig. 4. Reconstructed images using "phantom" mask: a) Haar; b) Haar_TI; c) CDF1.1; d) RBIO1.1.

The best results for the orthogonal wavelets and translation invariant variants are achieved by using the Haar, Daubechies2 or Symmlet1 wavelets. The worst result is achieved in the case of Daubechies50. The difference between the best and the worst case is of $1.75 \mathrm{~dB}$ in PSNR for orthogonal wavelets. When translation invariant is used, the difference between the best and the worst result is around $2 \mathrm{~dB}$. Biorthogonal wavelet that provides the best performance is CDF1.1 and RBIO1.1, and the worst one is Average-Interpolating. The best results from this phase of the experiment are achieved using translation invariant wavelets. The improvement is significant, more than $4 \mathrm{~dB}$ PSNR compared to the initial testing. Reconstructed images are shown in Fig. 4.

The third step of modification was applying doubledensity wavelets and using the generated sampling mask "phantom". The tests were performed for: Double-Density Discrete 2-D Wavelet Transform (S2DWT), Real 2-D Double-Density Dual-Tree DWT (R2DWT) and DoubleDensity Dual-Tree Complex 2-D DWT (C2DWT). The implementation of these wavelets was performed using the "Framelet2X" software package as a basis, developed as part of [10] - [12]. Other input parameters are kept the same as previously: number of iterations - 20, level of decomposition - 4 and sampling mask - "phantom". The results are shown in Table 3 and visual comparison between the reconstructed images can be seen in Fig. 5.

The experiment results on a phantom test image resonate to the results on the brain image [9]. The improvement of PSNR is $-0.55 \mathrm{~dB}$ and a small non-significant difference in SSIM and EPI results can be observed. The quantitative results for S2DWT and R2DWT are very close to each other.

TABLE 3: RECONSTRUCTION RESULTS USING "PHANTOM" MASK

\begin{tabular}{|c|c|c|c|c|}
\hline "phantom" & MAE & PSNR[dB] & SSIM & EPI \\
\hline S2DWT & 0.1942 & 35.5472 & 0.9451 & 0.9632 \\
\hline C2DWT & 0.2667 & 27.7022 & 0.5955 & 0.8827 \\
\hline R2DWT & 0.1810 & 35.2949 & 0.9392 & 0.9589 \\
\hline
\end{tabular}

In order to research the visibility of the artifacts and their influence in the process of forming a diagnosis, an expert of radiology was invited to assess the quality of images and potential clinical usage. After analyzing dozens of images, at different detail scale and different number of iterations for reconstruction and artifacts of these reconstructed images, his opinion was that S2DWT provided a good and reasonable quality compared to the original image.

\section{CONCLUSION}

In this paper a potential method for improving the techniques of compressed sensing and parallel imaging is proposed. The basis and reference for the performed research were already established software packages [7] [12].

POCS is chosen as a reconstruction method, not only for its simplicity, but also due to the fact that it outperforms other compared methods, such as zero-filled and GRAPPA [9]. The first conclusion is that for brain MRI scans, an elliptical sampling pattern would be the first choice, compared to the other forms of sampling masks. The conclusion could be expected, as the mask has a pseudorandom variable density of more samples in the center, with fewer samples on the edges. The mask has been generated using VDrad [8] software package already proven for other clinical purposes. We can also conclude that double-density wavelet transforms outperform the "traditional" wavelet families. This could also be expected taking into consideration that the number of samples is higher for these wavelets, as the name itself suggests. The usability of double-density wavelet transforms for clinical 
usage was also verified by a radiology expert. The results from the tests in this paper, confirm the results presented in [9].

This paper presents one possible direction for improving compressed sensing and parallel imaging. Future research for brain CS MRI may go towards: extended testing of the method, combination of several transforms, designing new more complex transforms, optimizing the elliptical sampling pattern and testing the proposed pair of sampling pattern and appropriate transform with other reconstruction methods.

\section{REFERENCES}

[1] Michael, Lustig, David, Donoho, Juan, Santos and John, Paulu, "A Compressed Sensing MRI.", IEEE Signal Processing, March 2008

[2] Michael, Lustig, David, Donoho and John, Paulu, "Sparse MRI: The Applicaton of Compressed Sensing for Rapid MR Imaging ", Magnetic Resonance in Medicine, December 2007

[3] F. Wajer, "Non-Cartesian MRI scan time reduction through sparse sampling," Ph.D. dissertation, Dept. Appl. Phys., Delft University of Technology, The Netherlands, 2001.

[4] Sergei Evladov, Ofer Levi and Adrian Stern, "Progressive compressive imaging from Radon projections", Optics express, 2012

[5] Lior Weizman and Yonina C. Eldar, "Reference-Based MR", Medical Physics Journal, 2016

[6] Christopher Adams Baker, "Investigation of Sparsifying Transforms in Compressed Sensing for Magnetic Resonance Imaging with Fasttestc", Ph.D. dissertation, 2016

[7] M. Lustig and J. Pauly, "SPIRiT: Iterative Self-Consistent Parallel Imaging Reconstruction from Arbitrary k-Space Sampling" MRM 2010;64(2):457-71

[8] Joseph Y. Cheng, Tao Zhang, Nichanan Ruangwattanapaisarn, Marcus T. Alley, Martin Uecker, John M. Pauly, Michael Lustig and Shreyas S. Vasanawala, "Free-Breathing Pediatric MRI with Nonrigid Motion Correction and Acceleration", Journal of Magnetic Resonance Imaging, August, 2015

[9] A. Kamilovski, "Sampling Mask and Wavelet Transform Experimental Testing for Improved Compressed Sensing of Brain MRI", Telfor 2018

[10] I. W. Selesnick, "The Double Density DWT," in Wavelets in Signal and Image Analysis: From Theory to Practice, A. Petrosian and F. G. Meyer, Eds. Boston, MA: Kluwer, 2001.

[11] I. W. Selesnick, "The Double-Density Dual-Tree DWT," in IEEE Transactions on Signal Processing, 52(5): 1304-14, May 2004.

[12] I. W. Selesnick and A. F. Abdelnour, "Symmetric Wavelet Tight Frames With Two Generators," Applied and Computational Harmonic Analysis, September 2004, Pages 211-225.

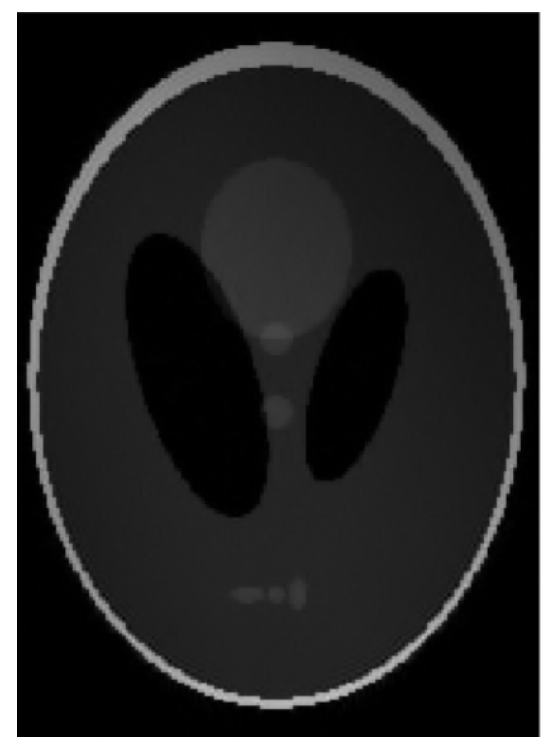

a)

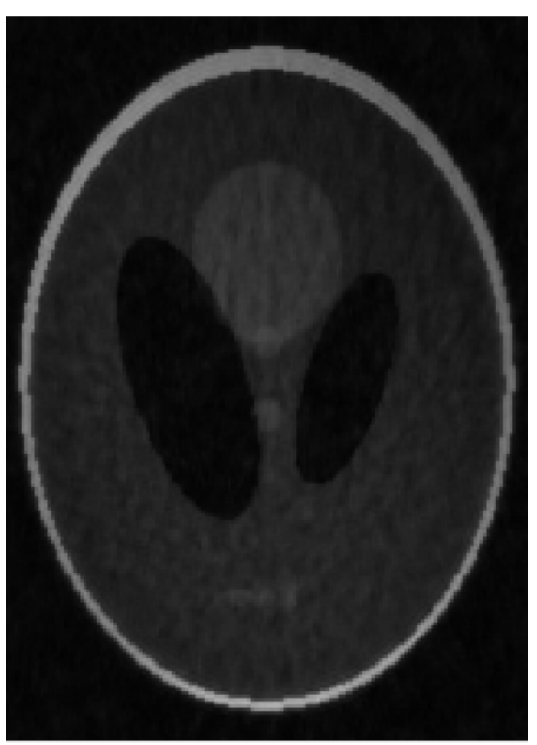

b)

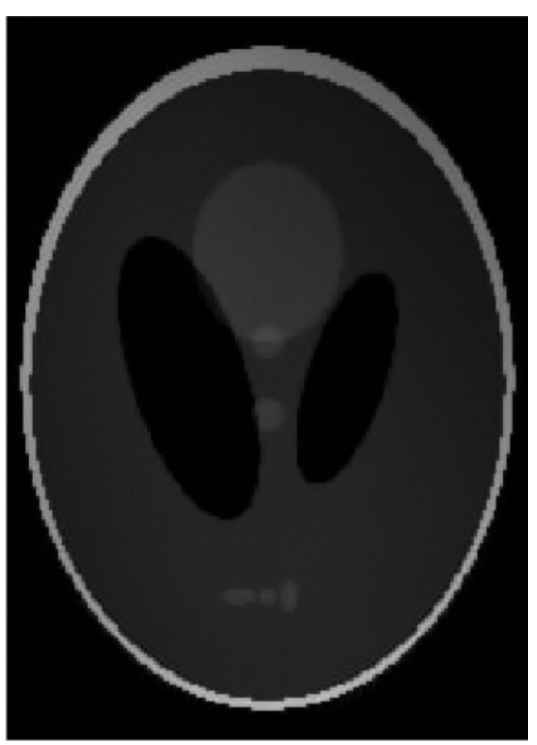

c)

Fig. 5. Reconstructed images using "phantom" mask: a) S2DWT; b) C2DWT; c) R2DWT. 